\title{
Sequential Liver Chemistry Profiling and Abdominal Ultrasound Assessments to Predict Biliary Strictures after Liver Transplantation
}

\author{
Kerem Sebib Korkmaz ${ }^{1}$, W. Rogier ten Hove ${ }^{1}$, Hein Verspaget ${ }^{1}$, Jeroen Dubbeld ${ }^{2}$, Ron Wolterbeek ${ }^{3}$, \\ Arian van Erkel ${ }^{4}$, Bert-Jan F. de Rooij ${ }^{1}$, Minneke C. Coenraad ${ }^{1}$, Jan Ringers ${ }^{2}$ and Bart van Hoek ${ }^{*}, 1$
}

\author{
Departments of Gastroenterology and Hepatology ${ }^{1}$, Transplantation Surgery ${ }^{2}$, Medical Statistics ${ }^{3}$ and Interventional \\ Radiology ${ }^{4}$, Leiden University Medical Center, Leiden, The Netherlands
}

\begin{abstract}
Background: After orthotopic liver transplantation (OLT) early detection of biliary strictures is important. Our aim was to evaluate the predictive value of routine serum liver chemistry profiling and abdominal ultrasound as noninvasive diagnostic tools in detecting biliary strictures after OLT.

Methods: We performed a retrospective study in which 141 primary OLTs, performed between 1992 and 2007 with more than 1 year follow-up, were included. Routinely assessed serum levels of alkaline phosphatase, alanine-aminotransferase, aspartate-aminotransferase, gamma-glutamyl transpeptidase and bilirubin at 3, 6, 9 and 12 months, and abdominal ultrasounds performed at 3,6 and 12 months after OLT were evaluated. All biliary strictures requiring intervention occurring after 3 months were included. Time-dependent Cox regression analysis was performed to identify predictive factors for the development of biliary strictures.

Results: Eighteen grafts developed non-anastomotic strictures (12.8\%) and 18 grafts (12.8\%) developed anastomotic strictures requiring intervention. An elevated gamma-glutamyl transpeptidase (HR 1.24 per $100 \mathrm{IU} / \mathrm{L} ; \mathrm{p}=0.05)$ and dilated bile ducts on ultrasound (HR 3.45; p < 0.01) were found to have an independent predictive value for the development of biliary strictures requiring intervention. Bilirubin and the other studied liver enzymes were not independently predictive.

Conclusion: Dilated bile ducts on ultrasound and elevated gamma-glutamyltranspeptidase after OLT are independent predictive factors for the development of biliary strictures requiring intervention. Routine assessment by serum gGT and US at 3-month intervals during the first year post-OLT is useful to screen for biliary strictures post-OLT.
\end{abstract}

Keywords: Liver enzymes, liver transplantation, strictures, time-dependent, ultrasound.

\section{INTRODUCTION}

Biliary complications are common after orthotopic liver transplantation (OLT), with a reported prevalence of $6 \%$ to $35 \%$ [1-4]. Biliary strictures occurring at the surgical anastomosis are classified as anastomotic strictures (AS), whereas strictures in the donor biliary tree are referred to as non-anastomotic strictures (NAS). Stricture formation is often insidious and usually only then detected when it leads to clinical symptoms as cholestasis, with serum liver enzyme abnormalities, intrahepatic bile duct dilatation and/or infection [5]. The definite diagnosis is made by endoscopic retrograde cholangiopancreatography (ERCP), percutaneous transhepatic cholangiography (PTC) or by magnetic resonance cholangiopancreatography (MRCP) [6, 7]. Although ERCP and PTC are considered the gold standard, they are invasive procedures and associated with considerable morbidity. ERCP and PTC are often immediately performed when clinical symptoms such as jaundice or cholangitis are present [8]. Several uncontrolled series evaluated the efficacy of ERCP in predicting biliary complications $[8,9]$. Most of

*Address correspondence to this author at the Department of Gastroenterology and Hepatology, C4-P, Leiden University Medical Center, PO Box 9600, 2300 ZC Leiden, The Netherlands; Tel: +31 (71) 5263507; Fax: +31 (71) 5248115; E-mail: B.van_Hoek@lumc.nl these were evaluated in settings where patients present themselves with symptoms such as cholangitis due to a biliary stricture. It is therefore important to detect early signs of stricture formation before such symptoms develop. Transplantation centers differ in how they routinely assess these problems. Liver biochemistry and abdominal ultrasound (US) may be useful for early detection of rejection, recurrence of primary disease (e.g., primary sclerosing cholangitis (PSC) or hepatitis) and bile duct pathology, such as stricture formation, since abnormalities may be present long before patients develop symptoms such as fever or abdominal pain. Only few studies addressed the predictive value of routinely assessed serum liver chemistry profiles and abdominal ultrasonography (US) after OLT as predictors for the occurrence of biliary strictures in a post-transplant population [10]. Although some studies did evaluate the prognostic value of liver chemistry and US, this was not in a time-dependent way and it remained unclear whether clinicians should routinely assess these diagnostic modalities in a post-transplant population for the early detection of biliary strictures, and at what time interval $[10,11]$. The risk of developing biliary strictures varies over time, probably in association with the liver chemistry profile and US findings. The aim of the present study was to evaluate the predictive value of routinely assessed serum liver chemistry and abdominal ultrasound as non-invasive predictors for the 
development of biliary strictures requiring intervention after OLT.

\section{PATIENTS AND METHODS}

\section{Patients}

We examined 141 consecutive first OLTs with at least one year of follow-up and complete data on serum liver chemistry and enzymes and upper abdominal ultrasonography (US) performed between September 1992 and April 2007 at the Leiden University Medical Centre. Re-transplantations $(n=31)$ were excluded. Clinical data were obtained from the medical digital records, the hepatological and surgical patient charts, and endoscopy reports. Follow-up was up to August 2008 with a median of 5.2 years (range 1.0 -15.6).

OLT was performed according to standard procedures with cavo-caval, porto-portal, and hepatic artery to hepatic artery anastomosis. A duct-to-duct biliary anastomosis over a 8-12 Ch stent was performed, if possible. The biliary stent was removed after 6 weeks or removed earlier as indicated. In some cases the hepatic artery was anastomosed to the aorta via an iliac conduit. All patients received immunosuppressive agents according to protocol: cyclosporin A or tacrolimus, prednisone during the first half-year and patients with renal impairment received azathioprine before 2001 or mycophenolate mofetil from 2001 on. From 2001 on, basiliximab was given post OLT. In some cases sirolimus was used after month 3 in which case the calcineurininhibitor was discontinued. All patients received ursodeoxycholic acid in the first 3 months after transplantation.

\section{Biochemical Variables}

Serum liver enzyme levels of alkaline phosphatase (ALP), alanine-aminotransferase (ALAT), aspartate-aminotransferase (ASAT) and gamma-glutamyltranspeptidase (GGT) were determined daily during the first two weeks and weekly for two months, after that at 3, 6, 9, and 12 months post-operatively. The same was done for bilirubin. Only the latter 4 time points were included in the study because in the first three months after liver transplantation liver enzymes are very susceptible to change due to procedure-related causes such as ischemia-reperfusion damage, early vascular complications such as hepatic artery thrombosis, rejection and infections. Therefore, the first three months of liver chemistry assessments, ultrasounds and biliary strictures after transplantation were excluded.

The upper limit of normal serum level was for ALP 120 IU/L, for ASAT, ALAT and GGT the upper limits of normal were $40 \mathrm{IU} / \mathrm{L}, 40 \mathrm{IU} / \mathrm{L}$ and $51 \mathrm{IU} / \mathrm{L}$, respectively. The upper limit for bilirubin (total) was $17 \mu \mathrm{mol} / \mathrm{L}$.

\section{Imaging Variables}

US was performed routinely on day 0,1 and 7 , and subsequently at 3,6 and 12 months after OLT. The US performed at 3,6 and 12 months were included in this study. These US were performed by different experienced radiologists. Due to lack of clear definition in the literature of dilated intrahepatic bile ducts a clear definition has been used by our radiologists with expertise in liver transplantation. In our institute a common bile duct of $>7 \mathrm{~mm}$ and an intrahepatic bile duct of $>2 \mathrm{~mm}$ on ultrasound were considered dilated and prompted either direct intervention by ERCP or PTC or additional MRCP which in turn might prompt ERCP or PTC. Other reported ultrasound findings of the biliary tree, such as sludge or a thickened biliary wall, were not taken into account in our analysis Routine abdominal CT scan was performed after 3 to 7 days post-OLT and routine liver biopsies were performed at 6 months after transplantation. Additional liver biopsies were taken on indication. Virology monitoring, including CMV-DNA, was performed frequently in the first year.

\section{Clinical Variables}

Presentation of a biliary stricture (anastomotic and nonanastomotic) was with clinical symptoms such as cholangitis, pruritus or jaundice and/or abnormal liver chemistry. Diagnostic tests to confirm the diagnosis were performed on indication but not included in the present analyses. Only strictures occurring more than three months after OLT that required intervention by ERCP, PTC or surgery were included in this study. From 2001 on, routine ERCP with stent-removal was performed at week 6 post-OLT in case of a duct-to-duct anastomosis. Accompanying the stent removal, a cholangiogram was also performed and possible strictures occurring at this time-point were excluded due to exclusion of liver enzymes and ultrasound findings in the first three months for the above mentioned reasons. All strictures were treated endoscopically with ERCP and dilation and/or stenting, and percutaneously with percutaneous transhepatic cholangiodrainage (PTCD) or with surgical intervention.

\section{Statistical Analyses}

We have used a time-dependent Cox regression model to evaluate the diagnostic value of liver enzymes and routine US assessments in predicting biliary strictures. The Cox proportional-hazards regression model for time-to-event data (e.g. the development of biliary strictures) takes into account the variable changes of variables over time, e.g. changes of liver enzymes and bile duct dilatation on US. Time dependent predictors (covariates) for stricture development in this study were liver chemistry variables ALP, GGT, ASAT, ALAT and bilirubin, obtained with an interval of three months at 3, 6, 9 and 12 months post OLT and US performed 3, 6 and 12 months post OLT. Recipient characteristics, like etiology of liver disease and procedure-related variables were baseline characteristics. Variables at a $p<0.20$ level in the univariate analysis were included in the multivariate analysis. Coefficients were considered significant when $p$ $<0.05$ in the multivariate analysis. The reported hazard ratios and p-values are per 100 international unit elevation for the liver enzymes. Bilirubin levels are reported per $10 \mu \mathrm{mol} / \mathrm{L}$ elevation.

\section{Ethical Committee}

All data were obtained as part of patient care according to a strict protocol after OLT. There was permission from the local ethics committee to use these data. 


\section{RESULTS}

\section{Patients and Biliary Strictures}

Baseline characteristics of recipients like etiology of liver disease and procedure-related variables are presented in Table 1. Non-anastomotic biliary strictures requiring intervention developed in 18 of the 141 grafts $(12.8 \%)$. Median time from OLT to NAS was 8.5 months (range 3-29). Median follow-up after the diagnosis of NAS was 5.5 years (range $0.0-11.6$ ). Anastomotic strictures developed in 18 out of 141 grafts $(12.8 \%)$. Median time from OLT to AS was 5.5 months (range 3-72). Median follow-up after the diagnosis of an anastomotic stricture was 2.8 years (range 0.6-15.3). Forty-one percent of the patients who developed a biliary stricture, both anastomotic and non-anastomotic, had biliary ducts dilatation on US whereas only $15 \%$ of the patients who had no biliary stricture requiring intervention had biliary ducts dilatation on US ( $p=0.001, \chi 2=11.65)$. Patients who developed a biliary stricture requiring intervention had a mean lag time of 5.3 months (standard error of the mean $(\mathrm{SEM})=0.75)$ from OLT to aberrant US whereas for patients who did not develop biliary strictures requiring intervention this was 6.2 months $(\mathrm{SEM}=0.85)$.

A total of twenty-one ERCPs and twelve PTCs for the management of strictures were performed in the included cases. In three cases a surgical intervention to resolve the stricture was necessary. There was no difference between the duct-to-duct type anastomosis and the Roux-en-Y anastomosis in the occurrence of biliary strictures $(\mathrm{p}=0.88)$. Other possible risk factors as primary liver disease, such as PSC,

Table 1. Baseline Characteristics of 141 Orthotopic Liver Transplants

\begin{tabular}{|c|c|}
\hline & N (141) \\
\hline Recipient data & $91 / 50$ \\
\hline Median age (years) (range) & $50(16-70)$ \\
\hline $\begin{array}{l}\text { Hepatitis B/C cirrhosis } \\
\text { Biliary cirrhosis (PSC/PBC) } \\
\text { Alcoholic cirrhosis } \\
\text { Hepatocellular carcinoma } \\
\text { Other }\end{array}$ & $\begin{array}{c}10 / 22 \\
30(22 / 8) \\
25 \\
19 \\
35\end{array}$ \\
\hline $\begin{array}{l}\text { Donor and OLT procedure data } \\
\qquad \begin{array}{c}\text { DBD / DCD donor } \\
\text { Choledochocholedochostomy (duct-to-duct)/ Roux-en-Y hepaticojejunostomy } \\
\text { Donor warm ischemic time (DCD donors) (minutes) (range) } \\
\text { Cold ischemic time (minutes) (range) } \\
\text { Recipient warm ischemic time (minutes) (range) }\end{array}\end{array}$ & $\begin{array}{c}135 / 6 \\
132 / 9 \\
17(11-23) \\
605(268-1095) \\
35(16-90)\end{array}$ \\
\hline
\end{tabular}

Table 2. Univariate Analysis of Potential Predictors for the Development of Biliary Strictures (BS)

Time-dependent analysis was used to calculate the predictive value of routinely performed liver chemistry profile assessments and dilated bile ducts on abdominal ultrasound (US) for detecting BS requiring intervention after OLT ( $\mathrm{n}=141)$. The hazard ratios for liver enzymes are shown per $100 \mathrm{IU} / \mathrm{L}$ increase. The hazard ratio for bilirubin is shown per $10 \mu \mathrm{mol} / \mathrm{L}$ increase.

\begin{tabular}{|c|c|c|}
\hline Clinical Variables & Hazard Ratio $(95 \%$ CI) & P-Value \\
\hline Dilated bile ducts on US & $4.48(1.97-10.12)$ & $<0.001$ \\
\hline ALP & $1.55(1.22-1.89)$ & $<0.001$ \\
\hline ALAT & $1.13(0.74-1.82)$ & 0.61 \\
\hline ASAT & $1.19(0.61-2.45)$ & 0.62 \\
\hline Gender & $0.64(0.33-1.40)$ & 0.23 \\
\hline Donation after cardiac death & $1.61(0.38-6.80)$ & 0.51 \\
\hline $\begin{aligned} \text { Underlying liver disease } & \\
& -\mathrm{PSC} \\
& -\mathrm{HCV} \\
& -\mathrm{PBC}\end{aligned}$ & $\begin{array}{c}0.04(0.00-18.72) \\
0.94(0.38-2.29) \\
1.13(0.27-4.73)\end{array}$ & $\begin{array}{l}0.31 \\
0.89 \\
0.87\end{array}$ \\
\hline Age (at OLT) & $0.97(0.95-1.00)$ & 0.14 \\
\hline
\end{tabular}


$\mathrm{PBC}$ and $\mathrm{HCV}$, or donation after cardiac death are also listed in Table 2.

\section{Liver Chemistry and Abdominal Ultrasound}

Elevation of serum liver enzymes ALP, GGT, ASAT and ALAT above the upper limit of normal occurred in respectively $57.4 \%, 71.6 \%, 53.9 \%$, and $61.0 \%$ of the patients at $3,6,9$ or 12 months after OLT. Bilirubin was elevated in $41.6 \%$ of the cases. There was a significant relationship between the level of GGT and the development of biliary strictures requiring intervention, both in the univariate and in the multivariate analysis (hazard ratios 1.35 and 1.25, p < 0.001 and $\mathrm{p}=0.04$, respectively), as presented in Tables 2 and 3. ALP above the upper limit of normal was also found to be a significant indicator for the development of biliary strictures in the univariate analysis $(p<0.001)$, but not in the multivariate analysis $(p=0.15)$. Elevated ALAT and ASAT were not associated with biliary strictures in the univariate analysis $(p=0.61$. and $p=0.62$ respectively). Elevation of bilirubin was not significant in both univariate and multivariate analysis for the prediction of the development of biliary strictures $(p=0.08$ and $p=0.32$ respectively (Table 3) Regarding the US assessments a significant relationship was found between dilated bile ducts on abdominal ultrasound and the successive development of a biliary stricture requireing intervention in both the univariate (hazard ratio $=4.48, \mathrm{p}$ $<0.001$ ) and multivariate analysis (hazard ratio $=3.45, \mathrm{p}<$ 0.01). (Table 3)

Table 3. Multivariate Time-Dependent Cox Regression Analysis for Liver Enzymes and Dilated Bile Ducts on Abdominal Ultrasound (US) for Detecting Presence of BS Requiring Intervention after OLT $(n=141)$

Gamma-glutamyltranspeptidase (GGT) and US remain significant predictors for the development of BS. The Hazard ratio for GGT shows the risk of having a stricture with each $100 \mathrm{IU} / \mathrm{L}$ increase. The hazard ratio for bilirubin was calculated for each $10 \mu \mathrm{mol} / \mathrm{L}$ increase.

\begin{tabular}{|c|c|c|}
\hline Clinical Variables & Hazard Ratio (95\% CI) & P-Value \\
\hline \hline Dilated bile ducts on US & $3.45(1.46-8.17)$ & $<0.01$ \\
\hline GGT & $1.24(1.00-1.54)$ & 0.05 \\
\hline ALP & $1.34(0.89-2.01)$ & 0.15 \\
\hline Bilirubin & $0.92(0.79-1.08)$ & 0.32 \\
\hline Age & $0.97(0.94-1.00)$ & 0.62 \\
\hline
\end{tabular}

\section{DISCUSSION}

Biliary strictures frequently complicate orthotopic liver transplantation and lead to significant morbidity, graft loss and mortality. Early diagnosis and prompt intervention is therefore of great clinical importance. Cholangiography remains the most sensitive and specific assessment in diagnosing biliary strictures but is invasive. The most commonly used and least invasive diagnostic modalities after OLT are serum liver chemistry profile determinations and abdominal ultrasound [12-15]. The prognostic values of abdominal ultrasound and liver enzymes in detecting biliary strictures have been evaluated before [16, 17]. Hussaini et al. [11], for example, showed that US was a valuable tool to diagnose biliary strictures with a sensitivity and specificity of $77 \%$ and $67 \%$, respectively. Que et al. [18] found US to detect biliary strictures with a sensitivity and specificity of $90 \%$ and $91 \%$ respectively, but reported GGT and ALP to be of poor diagnostic value even at 10 -folds the upper limit of normal. However, many transplantation centers still differ in the way they routinely assess liver biochemistry and US to detect biliary strictures and therefore different protocols for follow-up of transplanted patients are used. From previous studies it remained unclear whether routine assessment of these modalities can predict biliary strictures. In an outpatient clinic, patients often present themselves without symptoms, even if biliary strictures are present, but they have abnormal liver enzymes and US. Previous studies evaluated liver chemistry using sensitivity and specificity of US and liver enzymes in relation to the presence of biliary strictures. Although this is a common way to evaluate the diagnostic value of clinical tools it has several limitations: sensitivity and specificity only apply if the assessment of the liver biochemistry profile and biliary strictures occur simultaneously. Routine assessments of liver biochemistry and abdominal ultrasound in an outpatient clinic often precede the detection of a biliary stricture. In most previous studies sensitivity and specificity were based on dichotomized variables, e.g. liver enzymes were elevated or not. However, liver enzymes are continuous variables and change over time and probably concomitantly become elevated during stricture formation. This means that the risk of developing a biliary stricture for each patient varies along with the changes in liver enzymes or ultrasound findings. It is therefore more appropriate to use a time-dependent regression model for exploring predictive relationships by using quantities such as liver enzymes that vary over time [19]. The current findings are in accordance with non-transplantation studies in which GGT corresponds with the presence of biliary strictures, while mixed data are reported on the predictive value of liver chemistry for the presence of biliary complications after OLT $[8,10,12,18]$.

We found an independent association between the increased serum level of GGT assessed at fixed routine timepoints and the risk of detecting a biliary stricture requiring intervention. Time-dependent analysis calculates the hazard ratio for developing biliary strictures per $1 \mathrm{IU} / \mathrm{L}$ of elevated GGT, which in our study was 1.0022 . Thus, an elevation of $100 \mathrm{IU} / \mathrm{L}$ (i.e., $151 \mathrm{IU} / \mathrm{L}$ ) would result in $1.0022^{100}=1.24$ or a $24 \%$ increased risk. In formula terms, the hazard ratio for any elevation of GGT can be calculated $\left(1.0022^{\text {(elevation above }}\right.$ upper limit in $\mathrm{IU} / \mathrm{L})$ ) for the development of a biliary stricture. Abdominal ultrasound is a non-invasive, readily available and economic diagnostic tool. However, several studies observed that ultrasound is not very sensitive in detecting biliary strictures in a post transplant population, whereas few studies reported the opposite $[3,8,12,18]$. We found bile duct dilatation on abdominal ultrasound to be a powerful predictor of subsequent development of biliary strictures requiring therapy, exemplified by the high hazard ratio of 3.45 in the multivariate analysis. In clinical practice the calculated hazard ratios of both bile duct dilatation on US and the elevation of GGT are multiplied. In our example 
GGT levels $100 \mathrm{IU} / \mathrm{L}$ above the reference range together with dilated bile ducts on US result in a hazard ratio of 4.3 (3.45 x 1.24), which indicates that the risk of developing a biliary stricture requiring intervention is 4.3 more likely compared to the standard risk. Other modalities such as MRCP are also non-invasive tools which are widely used in diagnosing biliary strictures. However, MRCP is usually considerably more expensive than US and often has a waiting list, which makes it less applicable as a routine diagnostic tool. MRCP can be considered if other diagnostic tools provide no conclusive information and if there is less urgency in performing an ERCP. We realize that our study has some limitations. We decided to use a follow-up of one year after the 3-month time point since most strictures develop within the first year after OLT. Liver enzymes within the first three months were not included because early after transplantation many variables influence liver enzyme levels, such as ischemia-reperfusion damage, rejection and infection. A shorter follow-up would have weakened the statistical analysis. One should be careful using ultrasound findings which are not defined forehand. Unfortunately, there is no clear definition of dilated bile ducts in the literature and we therefore used a definition which is being used by our radiologist with expertise in bile duct dilatation and liver transplantation. Further prospective studies are needed to define a cut-off value for bile duct dilatation. Our timedependent regression analysis showed that detection of dilated bile ducts on US or elevated GGT are independent predictive factors for the development of biliary strictures requiring intervention in the first year after OLT. To our knowledge there are no other studies that evaluate the usefulness of routinely assessing liver chemistry and performing ultrasound after OLT. Routine assessment by serum GGT and US at 3-month intervals during the first year post-OLT is useful to screen for biliary strictures post-OLT. Elevated GGT or dilated bile ducts on US in the first year post-OLT should prompt cholangiography and may allow timely intervention before complications like cholangitis develop.

\section{CONFLICT OF INTEREST}

None declared.

\section{ACKNOWLEDGEMENT}

None declared.

\section{REFERENCES}

[1] Verdonk RC, Buis CI, Porte RJ, et al. Anastomotic biliary strictures after liver transplantation: causes and consequences. Liver Transpl 2006; 12(5): 726-35.
[2] Nishida S, Nakamura N, Kadono J, et al. Intrahepatic biliary strictures after liver transplantation. J Hepatobiliary Pancreat Surg 2006; 13(6): 511-6.

[3] Barriga J, Thompson R, Shokouh-Amiri H, et al. Biliary strictures after liver transplantation. Predictive factors for response to endoscopic management and long-term outcome. Am J Med Sci 2008; 335(6): 439-43.

[4] Pascher A, Neuhaus P. Bile duct complications after liver transplantation. Transpl Int 2005; 18(6): 627-42.

[5] Colonna JO, Shaked A, Gomes AS, et al. Biliary strictures complicating liver transplantation. Incidence, pathogenesis, management, and outcome. Ann Surg 1992; 216(3): 344-50.

[6] Morelli J, Mulcahy HE, Willner IR, et al. Long-term outcomes for patients with post-liver transplant anastomotic biliary strictures treated by endoscopic stent placement. Gastrointest Endosc 2003; 58(3): 374-9.

[7] Lee SH, Ryu JK, Woo SM, et al. Optimal interventional treatment and long-term outcomes for biliary stricture after liver transplantation. Clin Transplant 2008; 22(4): 484-93.

[8] Shastri YM, Hoepffner NM, Akoglu B, et al. Liver biochemistry profile, significance and endoscopic management of biliary tract complications post orthotopic liver transplantation. World J Gastroenterol 2007; 13(20): 2819-25.

[9] Shah SR, Dooley J, Agarwal R, et al. Routine endoscopic retrograde cholangiography in the detection of early biliary complications after liver transplantation. Liver Transpl 2002; 8(5): 491-4.

[10] Zoepf T, Maldonado-Lopez EJ, Hilgard P, et al. Diagnosis of biliary strictures after liver transplantation: which is the best tool? World J Gastroenterol 2005; 11(19): 2945-8.

[11] Hussaini SH, Sheridan MB, Davies M. The predictive value of transabdominal ultrasonography in the diagnosis of biliary tract complications after orthotopic liver transplantation. Gut 1999; 45(6): 900-3.

[12] Ben-Ari Z, Weiss-Schmilovitz H, Sulkes J, et al. Serum cholestasis markers as predictors of early outcome after liver transplantation. Clin Transplant 2004; 18(2):130-6.

[13] Zemel G, Zajko AB, Skolnick ML, et al. The role of sonography and transhepatic cholangiography in the diagnosis of biliary complications after liver transplantation. Am J Roentgenol 1988; 151(5): 943-6.

[14] Kok T, Van der Sluis A, Klein JP, et al. Ultrasound and cholangiography for the diagnosis of biliary complications after orthotopic liver transplantation: a comparative study. J Clin Ultrasound 1996; 24(3): 103-15.

[15] Uzochukwu LN, Bluth EI, Smetherman DH, et al. Early postoperative hepatic sonography as a predictor of vascular and biliary complications in adult orthotopic liver transplant patients. Am J Roentgenol 2005; 185(6): 1558-70.

[16] Li S, Stratta RJ, Langnas AN, et al. Diffuse biliary tract injury after orthotopic liver transplantation. Am J Surg 1992; 164(5): 536-40.

[17] Rull R, Valdecasas GJC, Grande L, et al. Intrahepatic biliary lesions after orthotopic liver transplantation. Transpl Int 2001; 14(3): 129-34.

[18] Que Y, Kaneko J, Sugawara Y, et al. Role of protocol ultrasonography for detecting biliary stricture in adult living donor liver transplantation recipients. Biosci Trends 2007; 1(1): 62-5.

[19] Kalbfleisch JD, Prentice RL. The statistical analysis of failure time data. New York: Wiley 1980. 